\title{
Predictor de mortalidad, utilidad de trauma score revisado. (RTS)
}

\author{
Mortality predictor, utility of revised trauma score. (RTS)
}

Jairo Israel Mayorga Mayorga. 1, Juan Israel Acurio Pérez. ², Fernando Sebastián Andrade Borja. ${ }^{3} \&$ Mónica Alexandra Ocaña Guevara. ${ }^{4}$

Recibido: 10-02-2020 / Revisado: 05-03-2020 /Aceptado: 22-03-2020/ Publicado: 04-04-2020

\begin{abstract}
.
DOI: https://doi.org/10.33262/concienciadigital.v3i2.1205
\end{abstract}

The leading cause of death and disability in the young adult population is trauma. Worldwide, an estimated 278.6 million people suffered trauma in special relation to traffic accidents. In Latin American countries, statistics are double compared to world power and that $20 \%$ to $40 \%$ of hospital occupation was related to this cause. The present investigation was carried out with the objective of assessment as a predictor of mortality on the Revised Trauma Score (RTS) scale in the emergency service of the General Teaching Ambato Hospital (HGDA). It was of an observation-documented nature with a qualitative and quantitative approach, of a non-experimental design, synthetic analytical and cross-sectional retrospective. It was defined that the variables of the vital signs were found in a normal percentage in most of the patients attended and in whom the calculation of the fuel scale could not be performed due to the limitation of the lack of information due to an incomplete volume of Clinically, in

\footnotetext{
${ }^{1}$ American College of Surgeons, Committee on Trauma, Advanced Trauma Life Support. Coordinación ATLS región 1 Quito, Ecuador. ATLS ID: 732052 Provider, jairomim@ @otmail.com

${ }^{2}$ Médico General de la República del Ecuador, Ambato, acuriojuan93@gmail.com

${ }^{3}$ Médico General de la República del Ecuador, Guaranda, fersab05@hotmail.com

${ }^{4}$ American Heart Association provider verify code: 795e59658435 Licenciada en enfermería, Riobamba, Ecuador, mony.aog12@gmail.com
} 
addition to pre-hospital care an triage, the implementation of RTS is needed to improve the time in care that influence the prognosis of these critically ill patients.

Keywords: Trauma Score Revised, mortality marker, triage, advanced trauma life support.

\section{Resumen.}

La principal causa de muertes y discapacidad en la población adulta joven es el trauma. A nivel mundial se estimó 278,6 millones de personas sufrieron traumas en especial relación con accidentes de tránsito. En países latinoamericanos las estadísticas se duplican versus con las potencias mundiales y que del $20 \%$ al $40 \%$ de la ocupación hospitalaria fue relacionada a esta causa. La presente investigación se realizó con el objetivo de valorar como predictor de mortalidad a la escala Trauma Score Revisado (RTS) en el servicio de emergencias del hospital General Docente Ambato (HGDA). Fue de carácter observacional-documentado de enfoque cuali-cuantitativo, de diseño no experimental, analítico sintético y de corte retrospectivo tranversal. Se definió que las variables de las constantes vitales se encontraron en un porcentaje normal en la mayoría de pacientes atendidos y en lis que no se pudo realizar el cálculo de la escala fue por la limitación de falta de información por un llenado incompleto de las historias clínicas, además que en la atención pre-hospitalaria como en el triage hace falta la implementación de RTS para mejorar el tiempo en la atención que influirá en el pronóstico de estos pacientes críticos.

Palabras claves: Trauma Score Revisado, marcador de mortalidad, triage, apoyo vital avanzado en trauma.

\section{Introducción.}

La constitución de la república del Ecuador en su Art 32 reza como obligación del estado ecuatoriano asegurar los derechos, sobre todo la salud, en fundamento de "equidad, interculturalidad, universalidad" En el año 2012 el ministerio de salud del Ecuador propuso un plan para mejorar el modelo de atención integral de salud con el fin de fortalecer la atención de emergencias en el sector extra hospitalario y hospitalario, dirigido a intensificar la misión de ofrecer el auxilio a las víctimas con el aprovisionamiento de bienes, abastecimiento y talento humano capaz(Ministerio de Salud Publica, 2014, citado en Mayorga, 2018)

En el plan nacional de desarrollo menciona al Art 280 y Art 293 como instrumento de partida de implementación de trabajo público enmarcadas para disminuir la miseria e inequidad para conseguir un gobierno de desarrollo y buen vivir. El vínculo de la salud con igualdad, de acceso libre sin marginación; por medio del incremento de profesionales de la salud, así como su aumento de cobertura y acceso.(Ecuador \& (CNP), 2017). Las prioridades de investigación de salud 2013-2017 se realizaron estudiando actores del sistema sanitario. Se reconocieron las áreas problemáticas con relación a determinantes individuales, ambientales y zoonoticos. 
Motivo por el cuál identificados los grupos etáreos vulnerables encontrados en el INEC (Instituto Nacional de Estadísticas y Censos) permiten emplear capitales e investigaciones para el progreso de los estándares óptimos de la terapéutica de traumatismos no intencionales y lesiones de trasporte.(Pública, Salud, \& Salud, 2013)

Los traumatismos es la primordial afección responsable de mortalidad en adultos jóvenes asimismo por causar gran porcentaje de discapacidad. La organización mundial de la salud (OMS), menciona que 278,6 millones de personas padecieron traumatismos. El 35\% fueron accidentes de tránsito,12,6\% caídas, 9,16\% violencia interpersonal.(Alberdi, García, Atutxa, \& Zabarte, 2014, citado en Mayorga, 2018)

Los países en vías de desarrollo el recuento de muertes en accidentes de tránsito tiene un valor duplicado al de países desarrollados.(Koshy, 2004). La OMS señala que la ocupación de camas hospitalarias es entre el $20 \%$ al $40 \%$ por personas que padecen traumas, necesitando mayor estadía hospitalaria para su recuperación y generando un consumo de recursos al sistema sanitario.(Hans Morten Lossius1, 2*, Marius Rehn1, 3, 1988, citado en Mayorga, 2018)

En la atención pre-hospitalaria ha surgido protocolos de adelanto de la calidad de atención, se cambió el paradigma de "cargar y correr" por el de "estabilizar y trasladar" brindando una atención adecuada por parte del servicio de socorro y clasificación de pacientes hacia la unidad de salud de destino apropiada para el tratamiento.(Villegas M1, Muñoz A1, 2017)

La herramienta para categorizar lesiones según la gravedad se la conoce como (Triage) usada ampliamente en los servicios de emergencia, la utilización inadecuada o el desconocimiento de esta produce un subtriage que causara la minimización del estado crítico de las lesiones, así mismo del sobretriage que destina más recursos de los necesarios. Según el colegio americano de cirujanos(ACS) el 5\% de subtriage y el 25\% al 50\% de sobre triage son permisibles.(Pallisera Lloveras, 2016)

Dentro de las escalas pronosticas para la clasificación de la gravedad en pacientes con trauma se destaca "Trauma Score Revisado" (RTS), realizando una evaluación simple y rápida de constantes vitales se obtiene resultados del $85 \%$ de sensibilidad, el rango de calificación es de 0 a 12 puntos; interpretada con mayor puntaje cuando existe un menor riesgo de letalidad y con menor puntaje a riesgo alto de muerte. El uso de esta escala mejora los tiempos de respuesta desde el diagnostico hasta el tratamiento cambiando el desenlace.(Jin, Shao, He, \& Yao, 2006, Champion, Sacco, Carnazzo, Copes, \& Fouty, 1981, citado en Mayorga, 2018)

En el trabajo publicado por el ministerio de salud pública del Ecuador en el año 2011, elaboró una serie de protocolos para la atención de profesionales en el área de emergencia prehospitalaria y hospitalaria por la carencia una guía de práctica (GPC) que norme el accionar en estas situaciones críticas.(Ecuador, 2011) 
Ecuador ocupó el segundo lugar de América Latina en el número de siniestros en las vías, información presentada en los reportes globales de la (OMS) del año 2013. El departamento de gestión de riesgos del hospital San Francisco de Quito en el mismo año determinó que de los accidentes viales fueron la sexta morbilidad de esta casa de salud y el $21,4 \%$ fueron lesiones severas, el 44,3\% moderados y el 34,3\% de lesiones menores.(Yánez Lucero, 2017, citado en Mayorga, 2018)

Del comité interinstitucional de red de emergencias médicas (CIREM), en el año 2017 "Villegas M" dijo que por no emplear (RTS) para la valoración de traumas surge una sobre estimación y sub estimación del triaje aplicado con estas personas afectando en sus probabilidades de subsistencia.(Villegas M1, Muñoz A1, 2017, citado en MAYORGA, 2018)

El instituto nacional de estadísticas y censos del Ecuador (INEC) en el año 2015, informó que por 100.000 ciudadanos 20 fallecen por circunstancias relacionadas con el tráfico.(Instituto Nacional de Estadistica y Censos, 2015)

En el país los decesos por accidentes de tráfico ocupan el séptimo lugar, dominando estas cifras los hombres con el $81 \%$ y de estos en atenciones pre-hospitalaria del $55 \%$ al $65 \%$ por traumatismos cráneo encefálicos.(Coordinación General de Planificación del MSP, 2016, citado en MAYORGA, 2018)

En síntesis (RTS) se creó como una herramienta para estratificar la severidad del trauma, el pronóstico y prioridad para el manejo integral de talento humano, recursos materiales y derivación oportuna ante la limitación de resolución.(Reyes, 2016, citado en MAYORGA, 2018)

\section{Antecedentes del trabajo}

Salvador F. Carlos en el año 2011, concluyo del uso de (RTS) en el hospital Luis Vernaza en pacientes poli-traumatizados en el área de emergencia, esta herramienta presentó un 85,5\% de sensibilidad y 91,7\% de especificidad siendo muy recomendable su implementación.(Dr. Carlos Luis Salvador Fernández, 2011,Citado En Mayorga, 2018)

Pallisera Ll. Anna en el año 2015, dijo que la mortalidad y los errores de la atención en traumas fueron por el retraso de tratamiento y referencia de pacientes críticos a unidades de mayor complejidad.(Pallisera Lloveras, 2016, citado en Mayorga, 2018)

Murillo L. Juan en el 2015, presentó un trabajo acerca de la aplicación del ATLS (Atención del Apoyo Vital Avanzado En Trauma) por sus siglas en inglés, en el hospital Roberto Calderón Gutiérrez, en el cuál expresa que la probabilidad de vida aumenta si se aplica el protocolo (ATLS).(Murillo López, 2016, citado en Mayorga, 2018)

Polo Z. Juan en el año 2016 hizo un estudio del (RTS) en una población de 68 pacientes con trauma grave con un valor del score de 5 puntos o menos, con el fallecimiento de estos casos 
se comprobó que la confianza en esta escala para la implementación en traumatismos.(Polo Zacarías, 2016, citado en MAYORGA, 2018)

Monar E. Andrea en el año 2016, en su tesis de grado señaló que en el hospital regional docente Ambato no cuenta con un sistema de evaluación para víctimas de lesiones que llegan al área de emergencia de esta unidad de salud.(Monar Espin Andrea Carolina;, 2014)

Cañizares N. Alejandra; Alejandra J. Lorena en el 2017 en su trabajo de tesis sobre el sistema de referencia de pacientes con poli-traumatismos en estado grave atendidos en el sistema ecu 911 hasta unidades de segundo y tercer nivel. Se aplicó (RTS) para la estratificación del criterio de gravedad y se obtuvo como resultado que, ante la necesidad de referencia a un hospital de tercer nivel, las persona no fueron llevados por el desconocimiento en dicha escala que puntúa criterios de severidad en la atención.(Cañizares N \& Altamirano J, 2017, citado en MAYORGA, 2018)

\section{(RTS) en la actualidad.}

Escala ocupada para pronosticar los traumas, integrada por tres variables fisiológicas. La falta de un marcador bioquímico es limitante por lo que se sugiere aumentar la variable ancho de distribución de glóbulos rojos (RDW). Se unió ambas herramientas (RTS+RDW) para crear RTS-R, una versión mejorada para calcular riesgos de muerte en 28 días.(Brown et al., 2018, citado en Mayorga, 2018)

\section{Concepto del trauma.}

El cambio morfológico estructural de manera abrupta o permanente en una persona accidentada se denomina trauma, producido por la liberación de energía sobre nuestro organismo.(Westhoff et al., 2003,citado en Mayorga, 2018)

\section{Menciones especiales a organizaciones internacionales.}

El comité de trauma del colegio americano de cirujanos (COT-ACS) menciona que las lesiones pueden variar en extensión y severidad poniendo en riesgo la vida cuando se tratan en un centro de trauma y piden concientización por la implementación del programa en toda la red de salud. (MacKenzie et al., 2006, citado en Mayorga, 2018)

En el trauma mayor según el comité de trauma (COT) su prioridad de tratamiento precoz es elevada porque de no hacerlo en minutos conllevan a la muerte de la víctima.(Lossius, Rehn, Tjosevik, \& Eken, 2012 citado en Mayorga, 2018)

\section{Fisiología.}

La lesión desencadena en el cuerpo un daño tisular del sistema cardiovascular y endotelial activando mediadores químicos de la inflamación o respuesta inflamatoria sistémica (SIRS), 
como efecto la respuesta compensadora mitiga el evento por anti-inflamación. Participa el sistema inmunológico específicamente las interleucinas (IL) continua la muerte celular y fallo multi-orgánico (FMO) terminando en la muerte.(Algattas \& Huang, 2013, citado en Mayorga, 2018)

La vasodilatación de la zona afectada inducida por la interleucina -6 (IL-6) estimula a los leucocitos para la creación de factor de necrosis tumoral (TNF) provoca inflamación y movilización de neutrófilos. Para combatir estos mecanismos se activan citosinas antiinflamatorias (IL-1Ram IL-4, IL-10, IL-11, IL-13).(Cañizares N \& Altamirano J, 2017)

Figura 1. Representación de mediadores Pro-Anti Inflamatorios

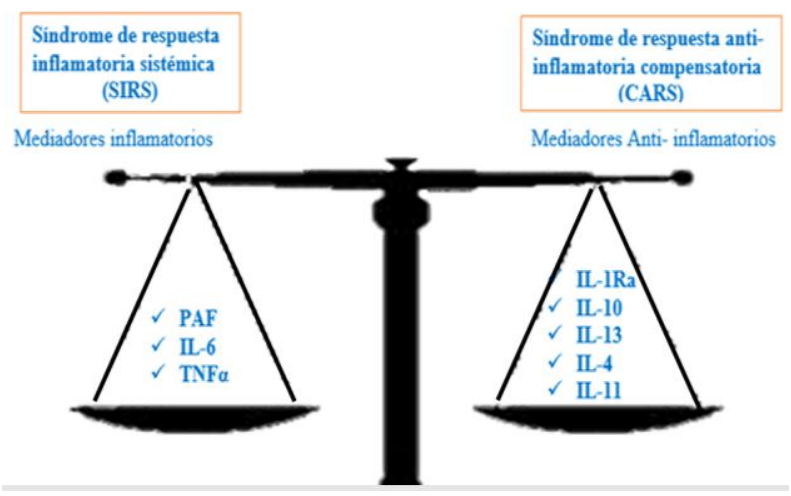

Fuente: A partir de (Cicchese et al., 2018, citado en MAYORGA, 2018).

\section{Clasificación del trauma.}

Tabla 1. Por la forma de presentación.

\begin{tabular}{cc}
\hline Abiertos & Cerrados \\
\hline $\begin{array}{c}\text { Con pérdida de } \\
\text { continuidad }\end{array}$ & $\begin{array}{c}\text { Excepto de perdida de } \\
\text { continuidad }\end{array}$
\end{tabular}

Fuente: A partir de(Cañizares N \& Altamirano J, 2017, citado en Mayorga, 2018)

Tabla 2. Traumas por su localización.

\begin{tabular}{|c|c|c|}
\hline \multicolumn{3}{|c|}{ Localización } \\
\hline Tipos & \multicolumn{2}{|c|}{ Valorar } \\
\hline \multirow{4}{*}{ Trauma torácico } & \multirow{4}{*}{$\begin{array}{l}\text { Problemas de circulación } \\
\text { Lesiones potencialmente }\end{array}$} & Obstrucción de la vía aérea \\
\hline & & Neumotórax a tensión \\
\hline & & Neumotórax abierto \\
\hline & & $\begin{array}{l}\text { Tórax inestable y contusión } \\
\text { pulmonar }\end{array}$ \\
\hline
\end{tabular}


ISSN: 2600-5859

Vol. 3, N², p. 34-54, abril-junio, 2020

Hemotórax masivo

Taponamiento cardiaco

\begin{tabular}{lll}
\hline $\begin{array}{l}\text { Trauma abdominal y } \\
\text { pélvico }\end{array}$ & $\begin{array}{l}\text { Penetrante } \\
\text { Cerrado } \\
\text { Por explosiones }\end{array}$ & $\begin{array}{l}\text { Peritoneo } \\
\text { Retro peritoneo } \\
\text { Pelvis } \\
\text { Periné }\end{array}$ \\
\hline & Severidad & \multicolumn{2}{c}{$\begin{array}{c}\text { Morfología } \\
\text { Trauma cráneo } \\
\text { encefálico }\end{array}$} & Leve & Intracraneal \\
& Moderado & \\
\hline & Severo & \\
Trauma raqui-medular & Nivel & \\
& Severidad del déficit neurológico \\
& Síndromes de la medula espinal \\
Mrauma musculo & Morfología & \\
esquelético & Hemorragia arterial & \\
& Amputación traumática & \\
& Fractura bilateral de fémur & \\
\hline
\end{tabular}

Fuente: A partir de(ATLS Subcommittee, American College of Surgeons' Committee on Trauma, \& International ATLS working group, 2013)

\section{Escalas de pronóstico.}

Creadas para mejorar las condiciones de los heridos brindando un diagnóstico precoz, tratamiento y estabilización oportuna, derivación a unidades de salud adecuadas que presten todas las necesidades clínico- quirúrgicas.(Polo Zacarías, 2016)

Tabla 3. Tipo de escalas pronosticas.

\begin{tabular}{ll}
\hline & \multicolumn{1}{c}{ Tipos } \\
\hline & Trauma Score (TS). \\
& Escala de Coma de Glasgow (ECG). \\
& RTS (Trauma score life support). \\
Fisiológicas & APACHE I, II y III. \\
& Índice de Trauma (Trauma Index TI). \\
& Prehospital Index. \\
& Abbreviated Injury Scale (AIS). \\
& Injury Severity Score (ISS). \\
& AP (Anatomic Profile o Perfil Anatómico). \\
& Organ Injury Scale (OIS). \\
& Escala de Lesión Orgánica \\
\hline
\end{tabular}




\begin{tabular}{ll} 
Combinadas & $\begin{array}{l}\text { CRAMS (Circulación, Respiración, Abdomen y } \\
\text { Tórax, Movimientos y Sonidos). }\end{array}$ \\
\hline $\begin{array}{l}\text { Probabilidad de } \\
\text { Supervivencia }\end{array}$ & $\begin{array}{l}\text { TRISS.(Trauma Injury Severity Score) } \\
\text { ASCOT (a Severity Characterization of Trauma). }\end{array}$ \\
\hline
\end{tabular}

Fuente: A partir de (Monar Espin Andrea Carolina;, 2014)

\section{Trauma score revisado.}

Califica al herido según constantes vitales (tensión arterial sistólica, frecuencia respiratoria, Glasgow).

Tabla 4. (RTS).

\begin{tabular}{|c|c|c|c|c|}
\hline (RTS) & 4 & 3 & 2 & 1 \\
\hline Glasgow & $15-13$ & 12-sep & 08-jun & 05-abr \\
\hline Tensión Arterial Sistólica & $>89$ & $89-76$ & $75-50$ & $49-1$ \\
\hline Frecuencia Respiratoria & 29-oct & $>29$ & 06-sep & $\begin{array}{l}01- \\
\text { may }\end{array}$ \\
\hline
\end{tabular}

Fuente: A partir de (Mayorga, 2010)

Tabla 5. Relación de puntaje con la supervivencia.

\begin{tabular}{cc}
\hline PUNTAJE (RTS) & \% \\
& SOBREVIVIENCIA \\
\hline 12 & $98-99$ \\
11 & $93-96$ \\
10 & $60-75$ \\
9 & $40-59$ \\
8 & $26-40$ \\
7 & $15-20$ \\
05-jun & $<10$ \\
01-abr & 0 \\
\hline
\end{tabular}

Fuente: A partir de.(Mayorga, 2010)

\section{Recepción del paciente.}

Tabla 6. Abordaje.

\begin{tabular}{ll}
\hline $\mathbf{M}$ & Mecanismo de lesión \\
\hline $\mathbf{I}$ & Injurias o lesiones encontradas \\
$\mathbf{S}$ & Signos y síntomas \\
$\mathbf{T}$ & Tratamiento indicado \\
\hline
\end{tabular}

Fuente: A partir de.(ATLS Subcommittee et al., 2013) 


\section{Historia clínica en trauma.}

Tabla 7. Anamnesis.

\begin{tabular}{ll}
\hline $\mathbf{A}$ & Alergias \\
\hline $\mathbf{M}$ & Medicamentos \\
$\mathbf{P}$ & Patologías y embarazo \\
$\mathbf{L i}$ & Libaciones, últimos alimentos \\
$\mathbf{A}$ & $\begin{array}{l}\text { Ambiente, escenario del } \\
\text { trauma }\end{array}$ \\
\hline
\end{tabular}

Fuente: A partir de.(ATLS Subcommittee et al., 2013)

\section{Revisión primaria.}

Tabla 8. Evaluación inicial.

\begin{tabular}{ll}
\hline A & Despejar vía aérea \\
\hline B & Ventilación \\
C & Circulación \\
D & Déficit neurológico \\
E & Exposición y control ambiental \\
\hline
\end{tabular}

Fuente: A partir de.(ATLS Subcommittee et al., 2013)

\section{A (vía aérea)}

Tabla 9. Miro, Escucho, Siento.

\begin{tabular}{ll} 
M & Boca, secreciones, cuerpos extraños, tráquea, yugulares \\
\hline $\mathbf{E}$ & Sonidos, estridor, ronquera \\
$\mathbf{S}$ & Alineación de la columna cervical
\end{tabular}

Fuente: A partir de.(ATLS Subcommittee et al., 2013)

\section{B (ventilación)}

Tabla 10. Miro, Escucho, Siento.

\begin{tabular}{ll}
\hline M & Expansibilidad torácica bilateral \\
\hline $\mathbf{E}$ & Murmullo vesicular presento o ausente \\
$\mathbf{S}$ & Mate o timpánico \\
\hline
\end{tabular}

Fuente: A partir de.(ATLS Subcommittee et al., 2013)

\section{C (circulatorio)}

Tabla 11. Control hemodinámico " $5 \mathrm{C}$ "

\begin{tabular}{ll}
\hline C & Calentar Lactato a $39^{\circ} \mathrm{C}$ en microondas \\
\hline C & Canalice 2 vías con catéter 14-16 \\
C & Cruce sangre, laboratorio, banco de sangre \\
C & Controle sangrado buscando sitio
\end{tabular}


C Cardiogénico, oír sonidos cardiacos

Fuente: A partir de.(ATLS Subcommittee et al., 2013)

\title{
D (deterioro neurológico)
}

Tabla 12. Evaluación neurológica

\author{
Pupilas \\ Glasgow \\ Focalidad neurológica

\begin{tabular}{ll}
\hline A & Alerta \\
\hline V & Responde estímulos verbales \\
D & Responde a estímulos dolorosos \\
I & Inconsciente \\
\hline
\end{tabular} \\ Fuente: A partir de.(ATLS Subcommittee et al., 2013) \\ E (exposición) \\ Tabla 13. Prevención de hipotermia (triada de la muerte)

\begin{tabular}{l} 
Manta caliente \\
\hline Rolling over
\end{tabular} \\ Fuente: A partir de.(ATLS Subcommittee et al., 2013)

\section{Metodología} \\ Se efectuó en el Hospital General Docente Ambato, en el servicio de emergencia; la \\ investigación se realizó a manera de un estudio de carácter observacional-documental de \\ enfoque cuali-cualitativo, de diseño no experimental, analítico-sintético, de corte \\ retrospectivo y transversal. Los datos se obtuvieron de la revisión de las historias clínicas de \\ pacientes (hoja 008), ingresados en esta área, con diagnóstico de traumatismo, dentro del \\ período establecido desde enero - abril 2018; mediante recolección de datos por sistema \\ informático Excel 2016 y luego llevados al software estadístico @ 2019 Minitab 19.1. Se \\ recogió un total de 80 pacientes atendidos en el servicio de emergencia de mencionado \\ hospital; se tomó como muestra a todo el universo de pacientes al ser un número menor de \\ 100 pacientes atendidos con el diagnostico mencionado, sujeto a criterios de selección. Se \\ determinaron los criterios de inclusión para el estudio, seleccionando a pacientes mayores de \\ edad vistos en mencionado servicio en el período enero-abril 2018 con diagnóstico de \\ Trauma. Se excluyó aquellas personas menores de edad, pacientes atendidos fuera del tiempo \\ establecido, pacientes vistas por otras causas al diagnóstico de trauma.
}

\section{Resultados.}

La tasa general de valores RTS [RR0(IC 95\%)11.704(12.081, 11.327); $\mathrm{p}=<0,005 \mathrm{~N}=27$ ]. La distribución de los valores de RTS son mayores en el puntaje de 12 existió un riesgo menor 
en la puntuación de la escala y en la historia clínica 00882 se produjo un fallecimiento a pesar del bajo riesgo encontrado.

Figura 2. Informe estadístico de resultados RTS

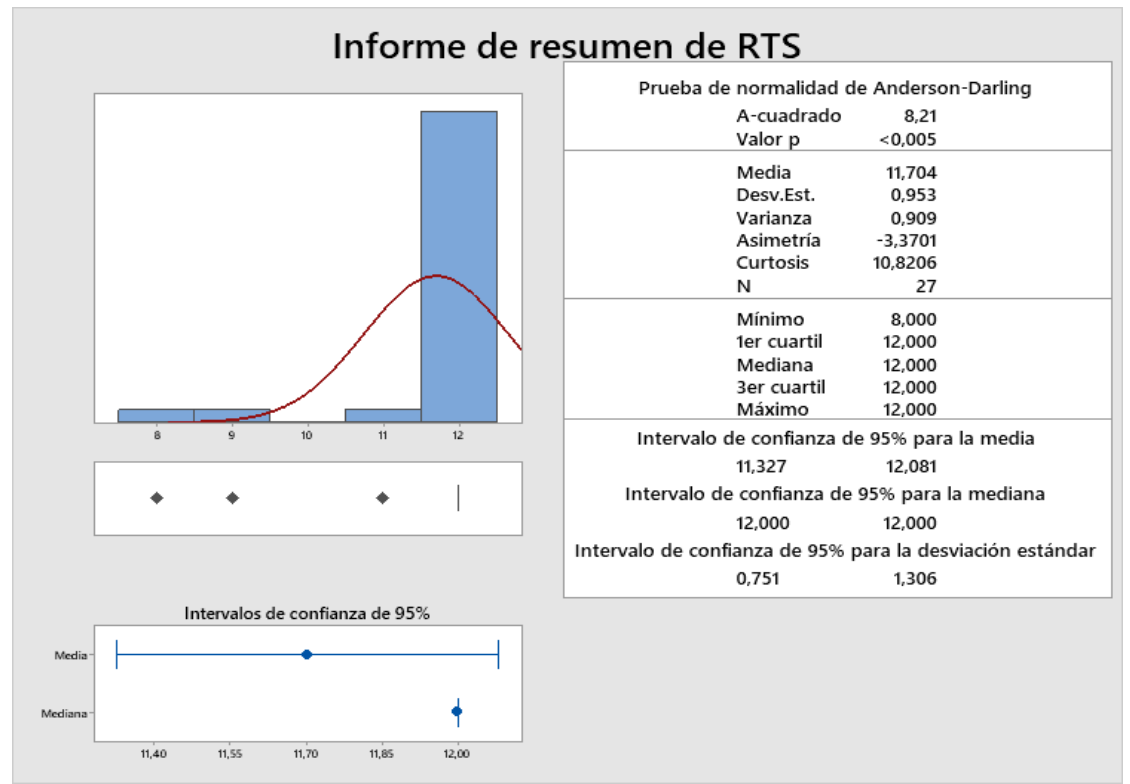

Fuente: Elaboración propia.

En un total de 27 historias clínicas recogidas con los datos suficientes para el cálculo de RTS se determino un valor de la Media $=11.70$, con un IC del 95\% para $\boldsymbol{\mu}$

\section{Estadísticas descriptivas}

Tabla 14. Medidas de asociación relacionadas a RTS.

\begin{tabular}{ccccc}
\hline N & Media & Desv.Est. & $\begin{array}{c}\text { Error estándar } \\
\text { de la media }\end{array}$ & $\begin{array}{c}\text { IC de 95\% } \\
\text { para } \boldsymbol{\mu}\end{array}$ \\
\hline 27 & 11,7 & 0,95 & 2,25 & $(7,29 ; 16,12)$ \\
\hline
\end{tabular}

$\mu$ : media de RTS

Desviación estándar conocida $=11,704$

Fuente: Elaboración propia.

La prueba se corrio con una $\boldsymbol{\mu}$ calculada del valor de la media $=10.25$ conseguida de los valores de las historias clínicas, la hipótesis nula se rechaza con un valor $\mathrm{p}=0,519$

\section{Prueba}

Tabla 15. Validación de hipótesis a RTS.

\begin{tabular}{ll}
\hline Hipótesis nula & $\mathrm{H}_{0}: \mu=10,25$ \\
\hline $\begin{array}{l}\text { Hipótesis } \\
\text { alterna }\end{array}$ & $\mathrm{H}_{1}: \mu \neq 10,25$
\end{tabular}




\begin{tabular}{ll} 
Valor $\mathbf{Z}$ & Valor $\mathbf{p}$ \\
0,65 & 0,519 \\
\hline
\end{tabular}

Fuente: Elaboración propia.

La tasa general de valores RTS [RR0(IC 95\%)39.441(34.733, 44.148); $=<0,005 \mathrm{~N}=59$ ]. La distribución de la edad indicó un mayor número de casos en la población adulta joven; como se describió en la bibliografía consultada e incluso coincidió con la historia clínica 0082 tuvo una edad de 29 años.

Figura 3. Informe estadístico de la edad en la poblacional estudiada.

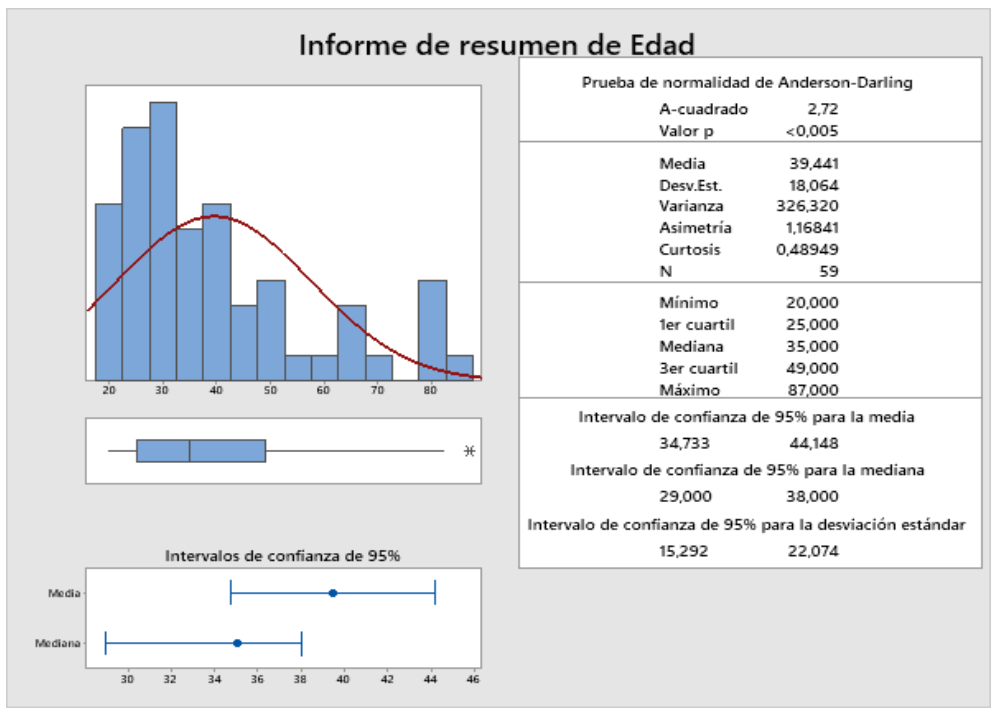

Fuente: Elaboración propia.

En un total de 59 historias clínicas recogidas con los datos necesarios para la determinación de la edad Media $=39.44$, con un IC del 95\% para $\boldsymbol{\mu}$

\section{Estadísticas descriptivas}

Tabla 16. Medidas de asociación relacionadas a la edad de la población de estudio.

\begin{tabular}{rrrrr}
\hline N & Media & Desv.Est. & $\begin{array}{c}\text { Error estándar } \\
\text { de la media }\end{array}$ & $\begin{array}{c}\text { IC de 95\% } \\
\text { para } \boldsymbol{\mu}\end{array}$ \\
\hline 59 & 39,44 & 18,06 & 2,35 & $(34,83 ; 44,05)$ \\
\hline
\end{tabular}

$\mu$ : media de Edad

Desviación estándar conocida $=18,064$

Fuente: Elaboración propia. 
La prueba se corrio con una $\boldsymbol{\mu}$ calculada del valor de la media=39.44 conseguida de los valores de las historias clínicas, la hipótesis nula se rechaza con un valor $\mathrm{p}=1,000$

\section{Prueba}

Tabla 17. Validación de hipótesis a la edad.

\begin{tabular}{ll}
\hline Hipótesis nula & $\mathrm{H}_{0}: \mu=39,441$ \\
\hline $\begin{array}{l}\text { Hipótesis } \\
\text { alterna }\end{array}$ & $\mathrm{H}_{1}: \mu \neq 39,441$ \\
Valor $\mathbf{Z}$ & Valor $\mathbf{p}$ \\
0 & 1 \\
\hline
\end{tabular}

Fuente: Elaboración propia.

La tasa general de valores RTS [RR0(IC 95\%)117.39(113.14, 121.64); $\mathrm{p}=<0,005 \mathrm{~N}=56$ ].

La distribución del valor de presión arterial sistólica se encontró dentro del rango adecuado y la historia clínica 0082 que tuvo un valor de $110 \mathrm{mmHg}$.

Figura 4. Informe estadístico de la presión arterial sistólica en la poblacional estudiada.

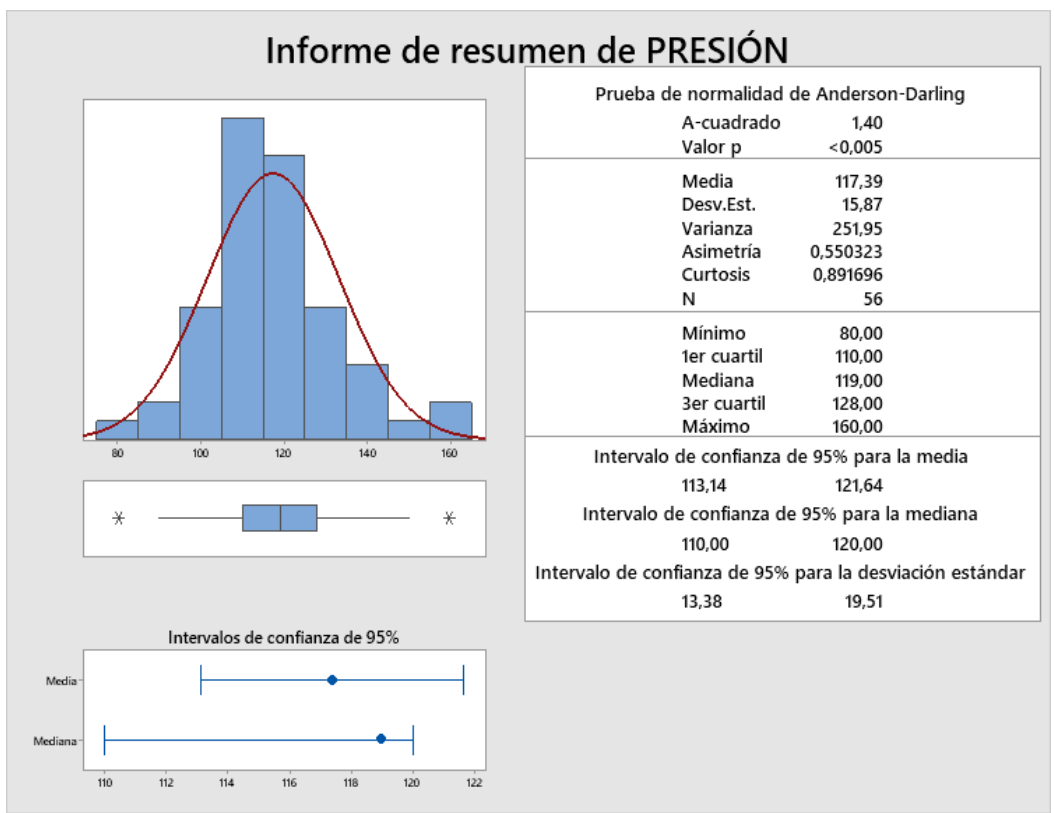

Fuente: Elaboración propia.

En un total de 56 historias clínicas recogidas con los datos necesarios para la determinación de la presión arterial sistolica Media=117.39, con un IC del 95\% para $\boldsymbol{\mu}$ 
Vol. 3, N², p. 34-54, abril-junio, 2020

\section{Estadísticas descriptivas}

Tabla 18. Medidas de asociación relacionadas a la tensión arterial sistólica de la población de estudio.

\begin{tabular}{ccccc}
\hline N & Media & Desv.Est. & $\begin{array}{c}\text { Error estándar } \\
\text { de la media }\end{array}$ & $\begin{array}{c}\text { IC de 95\% } \\
\text { para } \boldsymbol{\mu}\end{array}$ \\
\hline 56 & 117,39 & 15,87 & 2,12 & $(113,24 ; 121,55)$
\end{tabular}

$\mu$ : media de PRESIÓN

Desviación estándar conocida $=15,87$

Fuente: Elaboración propia.

La prueba se corrio con una $\boldsymbol{\mu}$ calculada del valor de la media=117,39 conseguida de los valores de las historias clínicas, la hipótesis nula se rechaza con un valor $\mathrm{p}=0,999$

\section{Prueba}

Tabla 19. Validación de hipótesis a la tensión arterial sistólica.

\begin{tabular}{ll}
\hline Hipótesis nula & $\mathrm{H}_{0}: \mu=117,39$ \\
\hline $\begin{array}{l}\text { Hipótesis } \\
\text { alterna }\end{array}$ & $\mathrm{H}_{1}: \mu \neq 117,39$ \\
Valor $\mathbf{Z}$ & Valor $\mathbf{p}$ \\
0 & 0,999 \\
\hline
\end{tabular}

Fuente: Elaboración propia.

La tasa general de valores RTS [RR0(IC 95\%)21.676(20.722, 22.631); $\mathrm{p}=<0,005 \mathrm{~N}=68$ ].

La distribución del valor de frecuencia respiratoria se encontró dentro del rango adecuado y la historia clínica 0082 que tuvo un valor de $24 \mathrm{rpm}$.

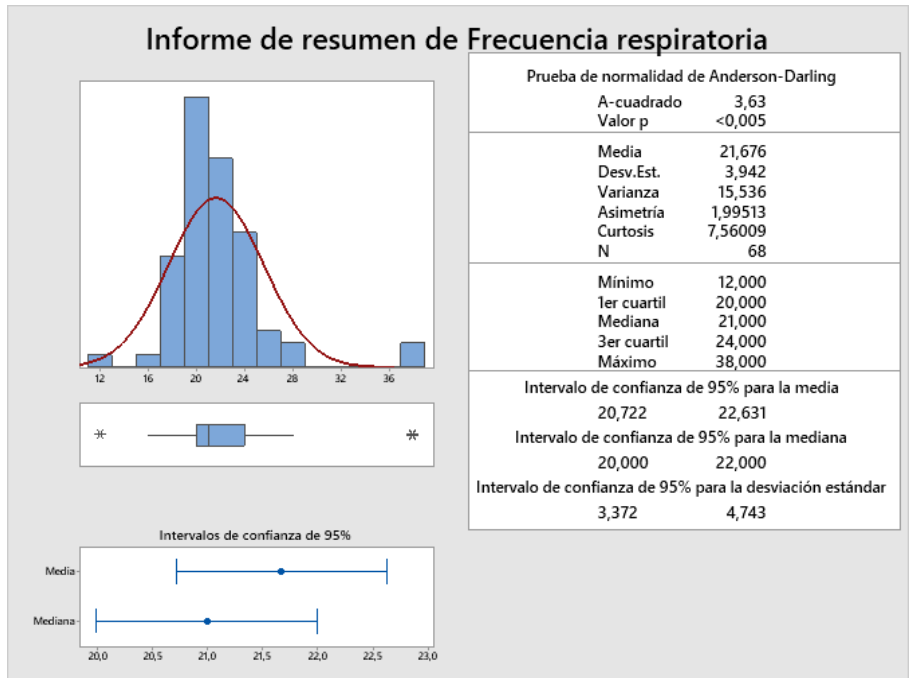

Figura 5. Informe estadístico de la frecuencia respiratoria en la poblacional estudiada. Fuente: Elaboración propia. 
En un total de 68 historias clínicas recogidas con los datos necesarios para la determinación de la frecuencia respiratoria Media $=21.676$, con un IC del 95\% para $\boldsymbol{\mu}$

\section{Estadísticas descriptivas}

Tabla 20. Medidas de asociación relacionadas a la frecuencia respiratoria de la población de estudio.

\begin{tabular}{ccccc}
\hline $\mathbf{N}$ & Media & Desv.Est. & $\begin{array}{c}\text { Error estándar } \\
\text { de la media }\end{array}$ & $\begin{array}{c}\text { IC de 95\% } \\
\text { para } \boldsymbol{\mu}\end{array}$ \\
\hline 68 & 21,676 & 3,942 & 0,478 & $\begin{array}{c}(20,740 ; \\
22,613)\end{array}$ \\
\hline
\end{tabular}

$\mu$ : media de Frecuencia respiratoria

Desviación estándar conocida $=3,942$

Fuente: Elaboración propia.

La prueba se corrio con una $\boldsymbol{\mu}$ calculada del valor de la media=21,676 conseguida de los valores de las historias clínicas, la hipótesis nula se rechaza con un valor $\mathrm{p}=0,999$

\section{Prueba}

Tabla 21. Validación de hipótesis a la frecuencia respiratoria.

\begin{tabular}{ll}
\hline Hipótesis nula & $\mathrm{H}_{0}: \mu=21,676$ \\
\hline Hipótesis & $\mathrm{H}_{1}: \mu \neq 21,676$ \\
alterna & Valor $\mathbf{p}$ \\
Valor $\mathbf{Z}$ & 0,999 \\
0 & \\
\hline
\end{tabular}

\section{Fuente: Elaboración propia.}

En un total de 77 historias clínicas recogidas con los datos necesarios para la determinación del lugar de donde se referenció el trauma, el cantón de Ambato fue el que obtubo el mayor porcentaje con un $74 \%$ de casos y la historia clínica 00882 pertencia a este grupo.

Figura 6. Informe porcentual de la cantidad de traumas según el sitio del evento en la poblacional estudiada.

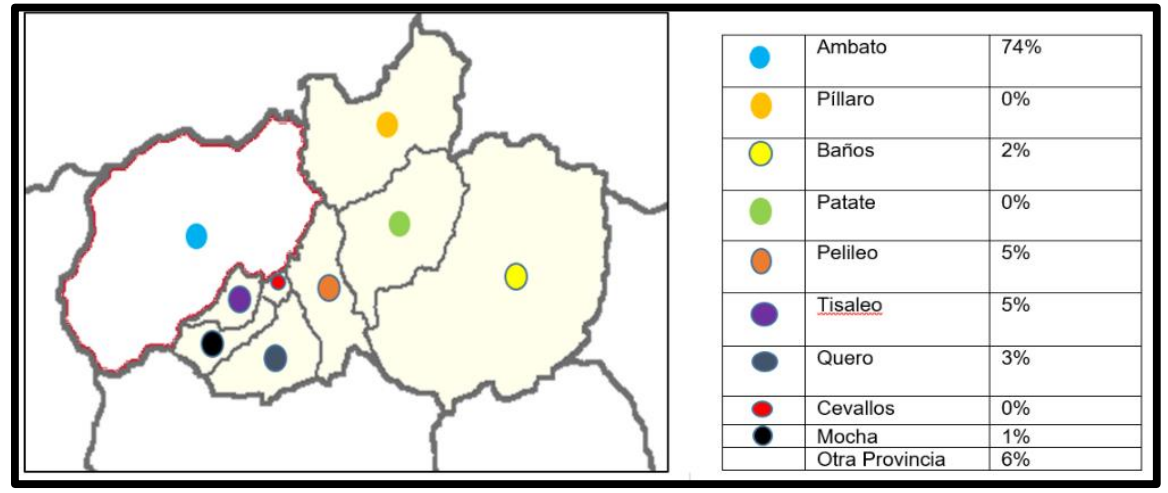

Fuente: Elaboración propia. 
En un total de 77 historias clínicas recogidas con los datos necesarios para la determinación del tipo de asistencia pre-hospitalaria a los pacientes, el 58\% de ellos acudió de manera espontanea por sus medios sin contar con una atención oportuna. La historia clínica 00882 pertenció al grupo que fue atendido por personal del Ecu 911.

Figura 7. Informe porcentual del tipo de asistencia pre-hospitalaria en la poblacional estudiada.

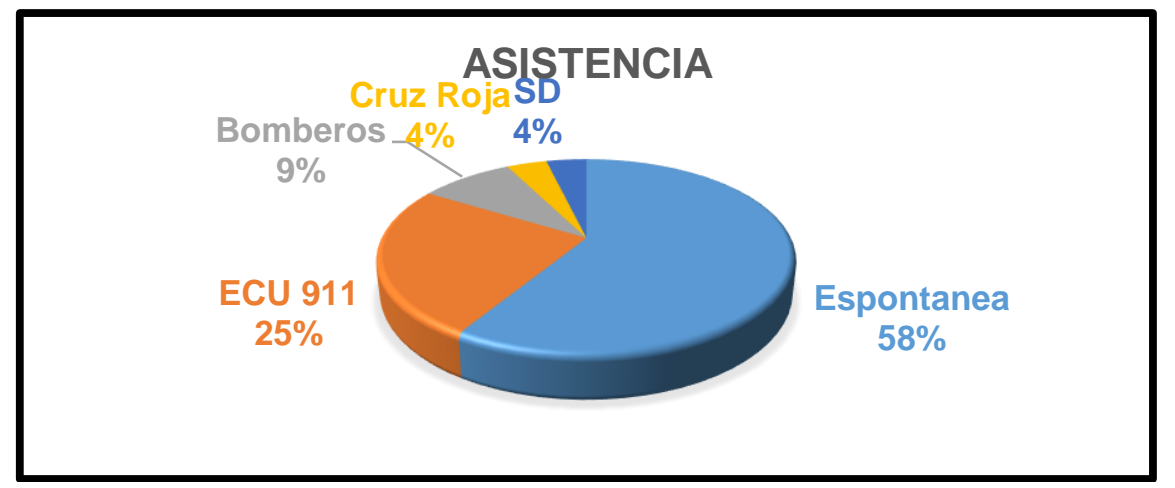

Fuente: Elaboración propia

En un total de 80 historias clínicas recogidas con los datos necesarios para la determinación del tiempo de incio de atención médica, el 53\% de ellos fue atendido en un tiempo mayor al recomendado en el protocolo descrito. La historia clínica 00882 pertenció al grupo que fue atendido en un tiempo adecuado.

Figura 8. Informe porcentual del tiempo de atención en la poblacional estudiada.

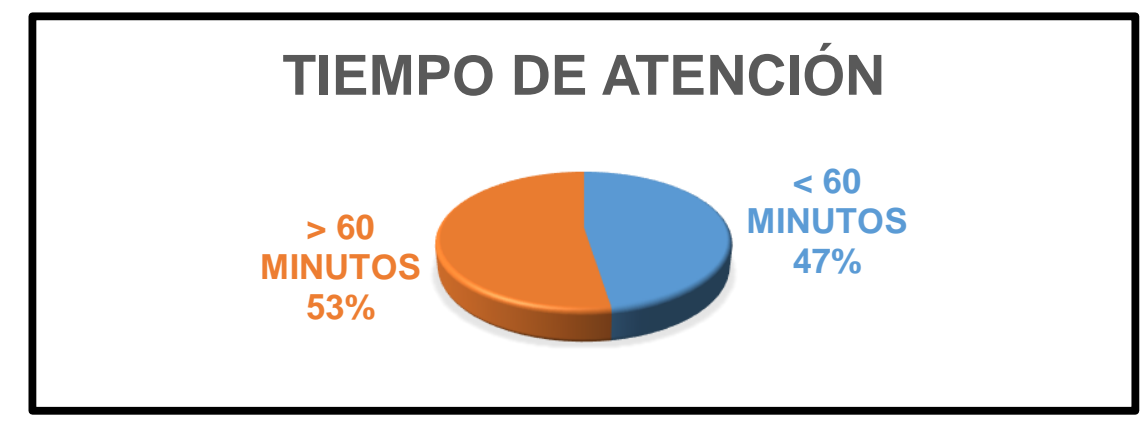

Fuente: Elaboración propia

En un total de 80 historias clínicas recogidas con los datos necesarios para la determinación de la mortalidad. La historia clínica 00882 fue el $1 \%$ de decesos en este grupo de estudio. 
Figura 9. Informe porcentual de la mortalidad en la poblacional estudiada.

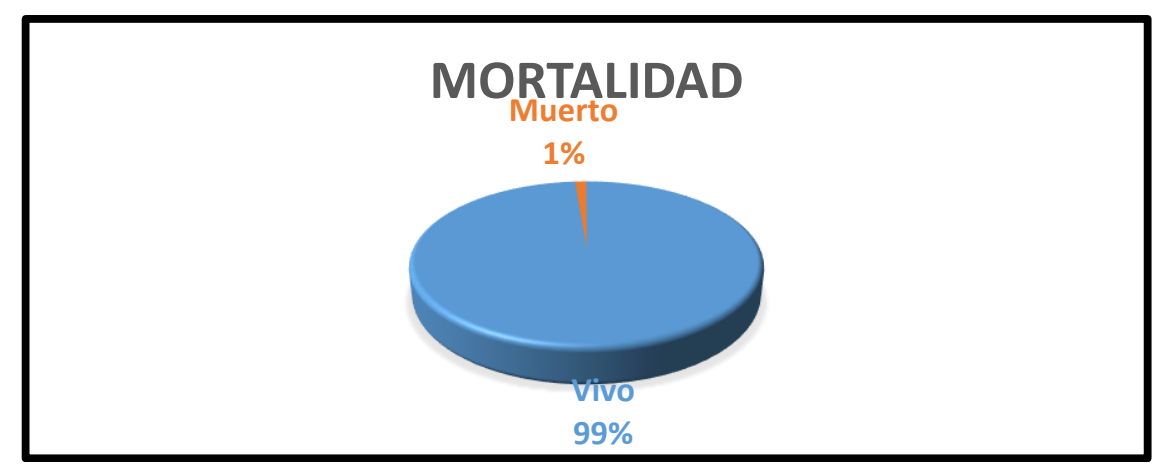

Fuente: Elaboración propia

\section{Conclusiones.}

- Se definió el marco legal del Ecuador para favorecer este campo de investigación y tratamiento, así como principios básicos de la patología para sugerir un protocolo inicial implementando (RTS) a la atención de pacientes con trauma.

- En la información recogida de las historias clínicas se encontró una limitante muy grande en el llenado, al no estar completa la información para la determinación de cada variable con sus riesgos inherentes.

- El resultado del cálculo de la escala (RTS) se obtuvo valores dentro de la normalidad en un mayor porcentaje teniendo relación con la baja mortalidad de la población estudiada.

- La asistencia pre-hospitalaria es fundamental para reducir el riesgo de muerte por trauma, en el estudio reveló que la mayor parte de la población acude a unidades hospitalarias por sus propios medios.

- La atención oportuna del trauma cambia favorablemente el desenlace, aproximadamente la mitad de la población de estudio fueron atendidos en un tiempo mucho mayor al esperado. Revelando la importancia del uso de (RTS) desde la atención pre-hospitalaria y triage.

\section{Referencias bibliográficas.}

Alberdi, F., García, I., Atutxa, L., \& Zabarte, M. (2014). Epidemiología Del Trauma Grave. Medicina Intensiva, 38(9), 580-588. Https://Doi.Org/10.1016/J.Medin.2014.06.012

Algattas, H., \& Huang, J. (2013). Traumatic Brain Injury Pathophysiology And Treatments: Early, Intermediate, And Late Phases Post-Injury. International Journal Of Molecular Sciences, 15(1), 309-341. Https://Doi.Org/10.3390/Ijms15010309

Atls Subcommittee, American College Of Surgeons' Committee On Trauma, \& International Atls Working Group. (2013). Advanced Trauma Life Support (Atls®). Journal Of Trauma And Acute Care Surgery, 74(5), 1363-1366. Https://Doi.Org/10.1097/Ta.0b013e31828b82f5 
Brown, L. E., Carroll, T., Lynes, C., Tripathi, A., Halperin, H., \& Dillon, W. C. (2018). Cpr Skill Retention In 795 High School Students Following A 45-Minute Course With Psychomotor Practice. The American Journal Of Emergency Medicine, 36(6), 11101112. Https://Doi.Org/10.1016/J.Ajem.2017.10.026

Cañizares N, A., \& Altamirano J, L. (2017). “Evaluación Del Sistema De Referencia De Pacientes Traumatizados Graves Desde El Área Prehospitalaria, Atendidos Por Unidades De Ambulancia, Integradas Al Ecu 911, Hacia Los Hospitales De Segundo Y Tercer Nivel Del Distrito Metropolitano De Quito, Desde J (Pontificia Universidad Católica Del Ecuador). Recuperado De Http://Repositorio.Puce.Edu.Ec/Bitstream/Handle/22000/12880/Tesis_Alejandra Cañizares_Lorena Altamirano.Pdf?Sequence $=1 \&$ Isallowed $=Y$

Champion, H. R., Sacco, W. J., Carnazzo, A. J., Copes, W., \& Fouty, W. J. (1981). Trauma Score. Critical Care Medicine, 9(9), 672-676. Https://Doi.Org/10.1097/00003246198109000-00015

Cicchese, J. M., Evans, S., Hult, C., Joslyn, L. R., Wessler, T., Millar, J. A., ... Kirschner, D. E. (2018). Dynamic Balance Of Pro- And Anti-Inflammatory Signals Controls Disease And Limits Pathology. Immunological Reviews, 285(1), 147-167. Https://Doi.Org/10.1111/Imr.12671

Coordinación General De Planificación Del Msp. (2016). Producción Estadística Msp 2006-2016. Recuperado De Https:/Www.Salud.Gob.Ec/WpContent/Uploads/2015/01/Producción-Estadística-2006-2016.Pdf

Dr. Carlos Luis Salvador Fernández. (2011). Salvador, C. (2011). “Valor Pronóstico De La Escala De Trauma Modificada (Rts) En Pacientes Politraumatizados Atendidos En El Servicio De Emeregncias Del Hospital General Luis Vernaza. Guayaquil. (Hospital General Luis Vernaza). Recuperado De Https://Hospitalalcivar.Com/Uploads/Pdf/Valor Pronostico.Pdf

Ecuador, R. Del, \& (Cnp), C. N. D. P. (2017). Plan Nacional De Desarrollo 2017-2021Toda Una Vida. Recuperado De Www.Planificacion.Gob.Ec

Ecuador, M. De S. P. (2011). Protocolos De Atencion Prehospitalaria Para Atenciones Medicas. En Protocolos (Vol. 1). Recuperado De Https://Aplicaciones.Msp.Gob.Ec/Salud/Archivosdigitales/Documentosdirecciones/Dn n/Archivos/Protocolos De Atención Prehospitalaria Para Emergencias Médicas.Pdf

Hans Morten Lossius1, 2*, Marius Rehn1, 3, K. E. T. And T. E. (1988). Calculating Trauma Triage Precision: Effects Of Different Definitions Of Major Trauma. The Journal Of Trauma: Injury, Infection, And Critical Care, 28(1), 87-90. Https://Doi.Org/10.1097/00005373-198801000-00012

Instituto Nacional De Estadistica Y Censos. (2015). Ficha Metodológica-Indicador Ods. Recuperado De Http://Www.Ecuadorencifras.Gob.Ec/Documentos/WebInec/Sistema_Estadistico_Nacional/Objetivos_Desarrollo_Sostenible_Ods/Objetivo_3 
/Meta_3.6/Indicador_3.6.1/Fm_Tasa_De_Mortalidad_Accidentes_Transito.Pdf

Jin, J. F., Shao, J. F., He, X. J., \& Yao, M. Q. (2006). Application Of Revised Trauma Evaluation Program In Emergency Treatment Of Multiple Injuries. Chinese Medical Journal, 119(5), 426-429. Https://Doi.Org/10.1097/00029330-200603010-00014

Koshy, A. V. (2004). Informe Mundial Sobre Prevención De Los Traumatismos Causados Por El Tránsito. Organización Mundial De La Salud. Recuperado De Https://Www.Who.Int/Violence_Injury_Prevention/Publications/Road_Traffic/World _Report/Summary_Es.Pdf

Lossius, H. M., Rehn, M., Tjosevik, K. E., \& Eken, T. (2012). Calculating Trauma Triage Precision: Effects Of Different Definitions Of Major Trauma. Journal Of Trauma Management \& Outcomes, 6(1), 9. Https://Doi.Org/10.1186/1752-2897-6-9

Mackenzie, E. J., Rivara, F. P., Jurkovich, G. J., Nathens, A. B., Frey, K. P., Egleston, B. L., ... Scharfstein, D. O. (2006). A National Evaluation Of The Effect Of TraumaCenter Care On Mortality. New England Journal Of Medicine, 354(4), 366-378. Https://Doi.Org/10.1056/Nejmsa052049

Mayorga, J. (2010). Universidad Regional Autónoma De Los Andes "Uniandes" Facultad De Ciencias Médicas Carrera De Medicina Proyecto De Investigación Previo A La Obtención De Titulo Médico Cirujano Tema. Recuperado De Http://Dspace.Uniandes.Edu.Ec/Bitstream/123456789/9258/1/Piuamed059-2018.Pdf

Mayorga Mayorga Jairo Israel. (2018). Valoración De La Escala Trauma Score Revisado Como Marcador De Mortalidad En Pacientes Atendidos Con Trauma En El Servicio De Emergencia Del Hospital General Docente Ambato.

Ministerio De Salud Publica. (2014). Histórica Inauguración En Tungurahua: 3 Centros De Salud Tipo B, Un Hospital Para Baños Y Un Área Repotenciada En El Hospital De Ambato - Ministerio De Salud Pública. Recuperado El 16 De Agosto De 2018, De Https://Www.Salud.Gob.Ec/Historica-Inauguracion-En-Tungurahua-3-Centros-DeSalud-Tipo-B-Un-Hospital-Para-Banos-Y-Un-Area-Repotenciada-En-El-Hospital-DeAmbato/

Monar Espin Andrea Carolina; (2014). Utilización De La Escala Triss En Pacientes Politraumatizados Que Acudan Al Servicio De Emergencias Del Hospital Provincial Docente Ambato, Comprendido Entre El Período Del 1 De Enero Al 30 De Marzo Del 2016. (Universidad Regional Autonoma De Los Andes). Recuperado De Http://Dspace.Uniandes.Edu.Ec/Bitstream/123456789/5609/1/Piuamed016-2017.Pdf

Murillo López, J. A. (2016). Aplicación Del Protocolo De Atención Del Apoyo Vital Avanzado En Trauma (Atls) En La Revisión Primaria Y Secundaria En Pacientes Politraumatizados Atendidos En El Servicio De Emergencia Del Hospital Roberto Calderón Gutiérrez Enero 2015-Diciembre 2015 (Universidad Nacional Autonoma De Nicaragua Unan - Managua.). Recuperado De Http://Repositorio.Unan.Edu.Ni/1586/1/61943.Pdf 
Vol. 3, N², p. 34-54, abril-junio, 2020

Pallisera Lloveras, A. (2016). Mortalidad Y Errores En La Atención Al Paciente

Politraumático.Estudio Prospectivo (Universidad Autónoma De Barcelona).

Recuperado De

Https://Ddd.Uab.Cat/Pub/Tesis/2016/Hdl_10803_382644/Apll1de1.Pdf

Polo Zacarías, J. C. (2016). Universidad Nacional De Trujillo Facultad De Medicina

Escuela De Medicina \&Quot;Score De Trauma Revisado Como Predictor De

(Universidad Nacional De Trujillo). Recuperado De

Http://Dspace.Unitru.Edu.Pe/Bitstream/Handle/Unitru/3608/Polozacarias_J.Pdf?Seque nce $=1$

Pública, M. De S., Salud, C. G. De D. E. En, \& Salud, D. De I. De La. (2013). Prioridades

De Investigación En Salud, 2013-2017. Recuperado De

Https://Www.Investigacionsalud.Gob.Ec/Wp-

Content/Uploads/2019/03/1_Líneas_De_Investigación_Priorizadas_Por_El_Ministeri o_De_Salud_Pública0670108001551892114.Pdf

Reyes, D. L. C. L. I. J. C. (2016). Revista Médica Electrónica. Revista Médica Electrónica Hospital Militar Docente Mario Muñoz Monroy. Matanzas Apuntes, 38(6), 910-915. Recuperado De Http://Scielo.Sld.Cu/Scielo.Php?Script=Sci_Arttext\&Pid=S168418242010000200015

Villegas M1, Muñoz A1, Q. E. (2017). Utilidad De Escalas De Evaluación Prehospitalaria Del Trauma Utility Of Prehospital Trauma Assessment Scales Villegas. Revista Científica Inspilip $V, 1,12$. Recuperado De Http://Www.Inspilip.Gob.Ec

Westhoff, J., Hildebrand, F., Grotz, M., Richter, M., Pape, H. ., \& Krettek, C. (2003). Trauma Care In Germany. Injury, 34(9), 674-683. Https://Doi.Org/10.1016/S00201383(03)00147-5

Yánez Lucero, J. M. (2017). Capacidad De Respuesta Del Personal De Emergencia Del Hospital San Francisco De Quito Del Instituto Ecuatoriano De Seguridad Social Frente A Un Evento Adverso De Víctimas En Masa Mediante La Ejecución De Simulacros En El Período Febrero Del 2017 - Mayo De (Pontificia Universidad Catolica De Quito). Recuperado De Http://Repositorio.Puce.Edu.Ec/Bitstream/Handle/22000/13302/Eventos En Masa.pdf?sequence $=1 \&$ isAllowed $=\mathrm{y}$ 


\section{PARA CITAR EL ARTÍCULO INDEXADO.}

Mayorga Mayorga, J. I., Acurio Pérez, J. I., Andrade Borja, F. S., \& Ocaña Guevara, M. A. (2020). Predictor de mortalidad, utilidad de trauma score revisado. (RTS). ConcienciaDigital, 3(2), 34-54. https://doi.org/10.33262/concienciadigital.v3i2.1205

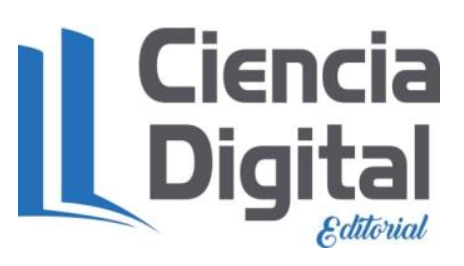

El artículo que se publica es de exclusiva responsabilidad de los autores y no necesariamente reflejan el pensamiento de la Revista Conciencia Digital.

El artículo queda en propiedad de la revista y, por tanto, su publicación parcial y/o total en otro medio tiene que ser autorizado por el director de la Revista Conciencia Digital.
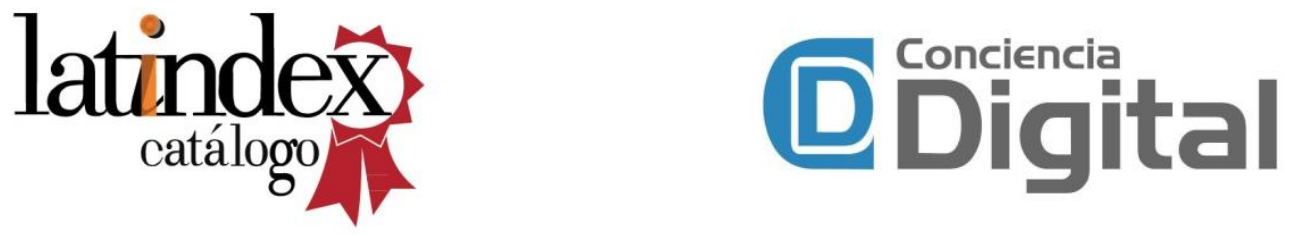